\title{
Hysteresis and relaxation of hard-soft nanocomposite samples
}

\author{
J. M. González ${ }^{\mathrm{a})}$ and M. I. Montero \\ Instituto de Ciencia de Materiales de Madrid-CSIC, Cantoblanco, 28049 Madrid, Spain \\ P. Crespo, P. Marín, and A. Hernando \\ Instituto de Magnetismo Aplicado, P.O. Box 155, 28230 Las Rozas, Madrid, Spain
}

\begin{abstract}
We present experimental results on the hysteresis and relaxation of $\mathrm{Fe} / \mathrm{Ba}$ hexaferrite composite samples prepared by ball milling starting from precursor Fe and Ba hexaferrite particles. Our results show that, for maximum applied fields of up to $8 \mathrm{kOe}$, the measured loops show a shift of up to 85 Oe. The compositional dependence of that loop displacement presents two maxima (at approximately $x=0.3$ and $x=0.7$ ) and a minimum at $x=0.5$. These results are discussed in terms of the dipolar coupling between both phases. (C) 2000 American Institute of Physics.
\end{abstract}

[S0021-8979(00)63708-8]

\section{INTRODUCTION}

It is widely recognized that the ubiquitous occurrence of exchange and dipolar interactions between the moments present in magnetically ordered materials influences both qualitatively and quantitatively their hysteretic and relaxational process. ${ }^{1-5}$ In the particular case of nanocrystalline, nanoparticulate, and nanocomposite materials, the potential for the tailoring of the extrinsic magnetic properties associated to the control of the interactions is specially relevant since in these materials the correlation lengths linked to the exchange and the dipolar coupling have magnitudes comparable to the morphological characteristic lengths which originate a broad range of hysteretic behaviors going from the extreme softness ${ }^{4}$ to the achievement of ultrahigh energy products in permanent magnet materials. ${ }^{3}$ Despite this, and the crucial technological relevance of the magnetization reversal-related properties, our control of the interactions is rather limited and should be improved in order to optimize the performance of the magnetic devices. There are, however, at least two relevant obstacles to overcome in order to achieve that goal: first, the absence of experimental techniques allowing us to measure the magnetic interactions at sufficiently short length scales ${ }^{6}$ and, second, the lack of a simple but sufficiently realistic description of the influence of the interactions on the reversal mechanisms (this last point is particularly relevant in the case of the dipolar interactions since those interactions are long ranged and have a manybody nature). In the present work we report on the hysteresis and relaxation of composite samples formed by nanosized $\mathrm{Ba}$ hexaferrite particles and micronsized $\mathrm{Fe}$ ones. As we will discuss below, those samples constitute an interesting model system to try to clarify the role of the magnetization reversal processes of the dipolar interactions.

\section{PREPARATION OF SAMPLES AND EXPERIMENTAL TECHNIQUES}

We have studied nanocomposite samples with nominal compositions given by $\mathrm{Fe}_{x} /\left(\mathrm{BaFe}_{12} \mathrm{O}_{19}\right)_{1-x}$, where $x$ de-

\footnotetext{
a) Also at: Instituto de Magnetismo Aplicado, PO Box 155, 28230 Las Rozas, Madrid, Spain; electronic mail: jesus.m.gonzalez@icmm.csic.es
}

noted a volume fraction and ranged from 0.1 up to 0.9. The samples were prepared by mixing in a ball mill (during 15 and $120 \mathrm{~min}$ ) nanoparticulate $\mathrm{BaFe}_{12} \mathrm{O}_{19}$ (average transmission electron microscope particle diameter $10 \mathrm{~nm}$, critical field $H_{\mathrm{cr}}=6.2 \mathrm{kOe}$ ) and Fe powders (average optical microscope particle size $45 \mu \mathrm{m}$, critical field $H_{\mathrm{cr}}=10 \mathrm{Oe}$ ). The precursor $\mathrm{Ba}$ hexaferrite particles were obtained by synthesis using a water-in-oil microemulsion. ${ }^{7}$ The structural and morphologic characterization of the samples involved x-ray diffraction and optical microscopy. The study of the magnetic properties of both the precursor materials and the composite samples was performed in press-powder cylinders $(3 \mathrm{~mm}$ height $\times 3 \mathrm{~mm}$ diameter) by using vibrating sample and superconducting quantum interference device magnetometers (maximum applied field $20 \mathrm{kOe}$ ).

\section{EXPERIMENTAL RESULTS}

The diffractograms taken in all the as-milled samples did not show any indication of the presence of phases different from the precursor ones. Particularly, the patterns corresponding to the samples with $x \geqslant 0.7$ exclusively exhibited $\alpha$-Fe reflections. Examination of the Fe-rich samples using an optical microscope (1 $\mu \mathrm{m}$ resolution) evidenced that the average particle sizes resulting from the milling process were $40 \mu \mathrm{m}$ (15 min milled samples) and $20 \mu \mathrm{m}$ (120 min milled samples), which led us to conclude that in these samples the $\mathrm{Ba}$ hexaferrite nanoparticles were fully embedded in the much larger $\mathrm{Fe}$ ones. Upon milling, the room temperature hysteresis loops of all the samples had smooth demagnetization branches indicating the occurrence of phase coupling. Figure 1 presents the low field data corresponding to a hysteresis loop measured with a maximum applied field of 8 kOe, in the $x=7,15$ min milled sample (the inset in Fig. 1 shows the complete loop). As it is apparent from Fig. 1, the loop was measurably displaced $(82 \mathrm{Oe})$ in the sense of the negative fields. That displacement was observed in all the samples, and its magnitude varied with the Fe volume fraction showing two maxima at, approximately, $x=0.3$ and $x$ $=0.7$ and a minimum at, approximately, $x=0.5$ (see Fig. 2). When a maximum field of $20 \mathrm{kOe}$ was applied, the loop displacements were not observed evidencing that the loops 


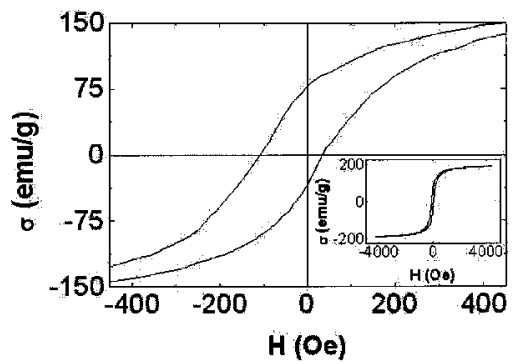

FIG. 1. Low field data corresponding to a hysteresis loop measured in the $x=0.7,15$ min milled sample with a maximum field of $8 \mathrm{kOe}$. The inset shows the complete loop.

measured under a maximum field of $8 \mathrm{kOe}$ were minor loops despite the fact that the maximum applied field was $30 \%$ higher than the critical field of the precursor $\mathrm{Ba}$ hexaferrite particles. In the inset in Fig. 2, we present the compositional evolution of the coercive force measured in the samples, which, with only the exception of the $x=0.1$ sample, was of the order of a few hundred oersted and much lower than the critical field of the $\mathrm{Ba}$ hexaferrite particles (in all these samples the coercivity was dominated by the high $\alpha$-Fe magnetization). An example of our results for the field evolution of the isothermal, $M_{i}$, and demagnetization, $M_{d}$, remanences is presented in Fig. 3 (where data corresponding to the $x=0.9,2 \mathrm{~h}$ milled sample are shown). $M_{i}$ was measured by increasing, starting from a demagnetized state, the maximum applied field up to $8 \mathrm{kOe}$ and $M_{d}$ was obtained by first submitting the sample to a (positive) magnetizing field of 8 $\mathrm{kOe}$ and then applying increasing demagnetizing (negative) fields down to $-8 \mathrm{kOe}$. In the inset in Fig. 3 we present the compositional evolution of the $\delta M=1-M_{d}(8 \mathrm{kOe}) /$ $M_{i}(8 \mathrm{kOe}) \mathrm{kOe}$ parameter (measuring the remanence asymmetry) which markedly decreased with the decrease of $x$ and went down to close to zero for $x=0.1$ (when both remanences were measured up to a maximum field of $20 \mathrm{kOe}$ the $\delta M$ values obtained in all the samples decreased to zero). Data corresponding to the time evolution of the demagnetization remanences measured at $T=10 \mathrm{~K}$, in the $x=0.7,2 \mathrm{~h}$ milled sample are shown in Fig. 4 (the sample was initially saturated by applying a magnetizing $20 \mathrm{kOe}$ field and then submitted to different demagnetizing fields which were, fi-

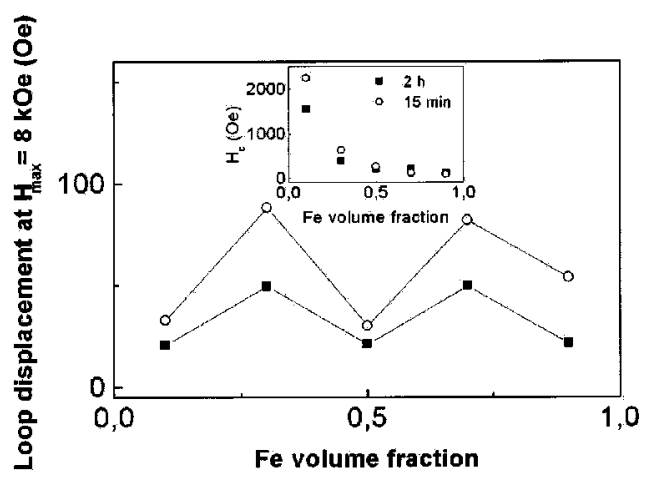

FIG. 2. Compositional evolution of the observed loop shift (maximum field $8 \mathrm{kOe}$ ). The inset presents the compositional variation of the coercivity of the samples.

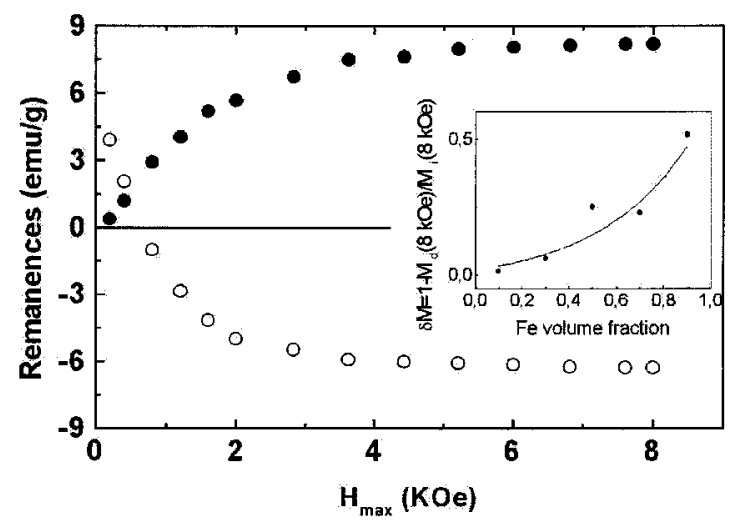

FIG. 3. Field evolution of the isothermal and demagnetization remanences ( $x=0.9,2 \mathrm{~h}$ milled sample). The inset shows the compositional evolution of the $\delta M$ parameter.

nally, decreased to zero for the recording of the time evolution of the magnetization). Our results show that for the fields below the coercive force of this sample the relaxation proceeded monotonously. Differently from this (see the inset), when the demagnetizing field was of the order of the remanent coercive force (300 Oe at the measurement temperature) it was possible to observe a nonmonotonous time evolution of the magnetization.

\section{DISCUSSION}

It is possible to rule out the relation of the observed minor loop displacement to the occurrence of any (oxide induced) exchange anisotropy. In the case of the Fe-rich samples, this is due to the large size of the Fe particles (always greater than $20 \mu \mathrm{m}$ ) that allows the low field reversal of the $\alpha$-Fe even if an oxide layer, originating that unidirectional anisotropy, were present at the particle surfaces (including the $\mathrm{Fe}-\mathrm{Ba}$ hexaferrite interfaces). Let us also remember that the loop shift linked to exchange anisotropy should be observable in major loops (which is not the case in any of the samples). For the discussion of our results we will consider two different compositional regions, above $(\mathrm{Ba}$ hexaferrite-rich samples) and below (Fe-rich samples) the $\mathrm{Ba}$

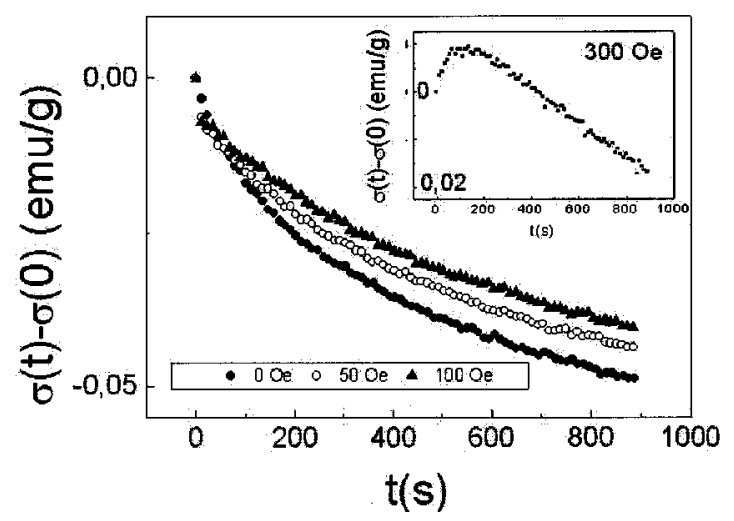

FIG. 4. Time dependence of the demagnetization remanences corresponding to different negative fields. The inset shows data corresponding to a demagnetizing field of $300 \mathrm{Oe}$ (measurements taken at $T=10 \mathrm{~K}$ in the $x=0.7,2 \mathrm{~h}$ milled sample). 
hexaferrite percolation concentration, respectively. In the $\mathrm{Fe}$ rich region, and due to the macroscopic continuity of this soft phase and the low measured coercivities, we propose the occurrence of a nucleation-propagation demagnetization mechanism. Thus, the soft phase reversal field, which approximately coincides with the global coercivity due to the high Fe magnetization, can be expressed as the sum of the coercivity of the precursor Fe particles and of a dipolar field (acting in the nucleation region) originated by the unreversed (and still having moments pointing close to the saturation direction) Ba hexaferrite nanoparticles. That dipolar field always points along the same sense and direction and we will thus identify it with the loop displacement. The Ba hexaferrite particles can create a nonzero dipolar field due to two different reasons: (i) their anisometry ${ }^{8}$ (that is, the fact that the $\mathrm{Ba}$ hexaferrite platelets have a demagnetizing factor close to $4 \pi$ whereas the approximately spherical Fe particles have a demagnetizing factor of the order of $4 \pi / 3$ ) and (ii) their nonhomogeneous spatial distribution (a perfect spherical distribution of hexaferrite particles should not originate any dipolar field, but in our samples the occurrence of disorder is apparent from the characteristics of the preparation method and, more clearly, from the observed anomalous relaxation taking place at remanences corresponding to demagnetization fields of the order of the coercivity, which evidence the presence in the samples of two types of regions at which the internal field can be mutually antiparallel).

To discuss the compositional dependence of the dipolar (loop displacement) field let us recall that, for low Ba hexaferrite concentrations, it should increase with the increase of the concentration of hard particles. Nevertheless, the dipolar field increase linked to the increase of the hexaferrite concentration competes, for sufficiently large concentrations, with the formation of agglomerates (involving that of fluxclosing particle structures). Since the density of agglomerates increases up to the percolation concentration, the experimentally observed maximum on the compositional dependence of the loop displacement reflects the convolution of the two mentioned mechanisms. Considering now the Ba hexaferrite-rich compositions, and as it has been evidenced in other mechanically hard-soft ( $\mathrm{Ba}$ hexaferrite and $\mathrm{Fe}$, re- spectively) composite systems obtained by ball milling, ${ }^{9}$ we will accept that the microstructure consists of small Fe particles embedded in the Ba hexaferrite matrix. Therefore, and although the coercivity mechanism of these Fe particles could in general be different from the nucleation-propagation one considered in the case of the Fe-rich samples the above discussion holds (now the dipolar field is associated to the nonhomogeneous distribution of Fe particles whose increase in number originates an increase of the dipolar field and whose agglomeration reduces the coercivity possibly through the formation of agglomerates able to accommodate domain walls). Let us also say that the small loop displacement observed in the $x=0.1$ sample should be influenced by the large contribution to the total magnetization of the sample of the $\mathrm{Ba}$ hexaferrite and that the minimum $(x=0.5)$ of the loop displacement should result from both the change of coercivity mechanisms and the large number of particles that can contribute to the dipolar field for these compositions (since it is related to the deviations from the perfect spherical particle distribution the magnitude of the dipolar field should be proportional to the reciprocal of the square root of the total number of particles contributing to the field). In order to confirm our description of the mechanisms underlying the presented phenomenology we currently proceed to the measurement of the hysteretic and relaxational properties of our samples at different temperatures and to development of a simple quantitative model of their behavior.

${ }^{1}$ G. Herzer, IEEE Trans. Magn. 26, 1397 (1990).

${ }^{2}$ T. Schrefl, H. Roitner, and J. Fidler, J. Appl. Phys. 81, 5567 (1997).

${ }^{3}$ J. M. D. Coey, J. Magn. Magn. Mater. 140-144, 1041 (1995).

${ }^{4}$ J. M. Alameda, L. T. Baczewski, B. Dieny, D. Givord, J. M. Ndjaka, J. P. Nozières, J. J. Préjean, J. P. Rebouillat, and F. H. Salas, J. Magn. Magn. Mater. 104-107, 1813 (1992).

${ }^{5}$ M. V. P. Altoé, M. S. Lancarotte, H. R. Rechemberg, F. P. Missell, and J. M. González, IEEE Trans. Magn. 31, 3614 (1995).

${ }^{6}$ C. de Julián, M. Emura, F. Cebollada, and J. M. González, Appl. Phys. Lett. 69, 4251 (1996).

${ }^{7}$ D. A. Rawlinson and P. A. Sermon, J. Phys. IV 7, 755 (1997).

${ }^{8}$ M. I. Montero, F. Cebollada, M. P. Morales, J. M. González, and A. Hernando, J. Appl. Phys. 83, 6277 (1998).

${ }^{9}$ A. K. Giri, C. de Julián, and J. M. González, J. Appl. Phys. 76, 6573 (1994). 